\section{Verursacht Känguruhing fetale Arrhythmien?}

Die Känguru-Methode ist auf allen neonatologischen Stationen fester Bestandteil der entwicklungsfördernden Pflege Früh- und Neugeborener. Das neonatologische Team der Universitätsklinik von Santiago de Compostela machte dabei eine interessante Beobachtung.

$B_{\text {eim }}^{\text {eis }}$ eim Känguruhing wird das nackte Baby auf den ebenfalls nackten Oberkörper der Mutter oder des Vaters gelegt. In einer Neugeborenenintensivstation in Spanien trat bei einem zwei Wochen alten Zwillings-Frühgeborenen der 32. Schwangerschaftswoche mit noch selbstlimitierenden Bradykardie-Episoden und ohne Atemhilfe während des Känguruhings plötzlich ein EKG-Alarm auf. Der Neonatolge registrierte eine Tachykardie mit einer Herzfrequenz über 200 pro Minute mit zwei überlagerten und grundverschiedenen EKG-Vektoren und -Rhythmen. Haut und Atmung des Mädchens waren jedoch normal. Das Pulsoxymeter registrierte eine regelmäßige Frequenz von nur 150 pro Minute. Mit dem Zurücklegen des Kindes in sein Wärmebett „sprang“ die Herzfrequenz im EKG des Kindes sofort wieder in einen normalen Sinusrhythmus um.

Lopez-Abel B et al. Kangaroo mother care induced arrhythmia. Klin Padiatr 2015;227:299-300

\section{Kommentar}

Bei diesem Phänomen handelt es sich um eine überlagerte elektrische Herzaktivität der Mutter während des engen Haut-zuHaut-Kontakts. Die Autoren beschreiben dies als mögliche Fehlerkennung in der EKG-Aufzeichnung. Weitere Untersuchungen müssen erst noch bestätigen, dass die Pulsoxymetrie eine weniger fehleranfällige Monitorüberwachung als das EKG-Monito-

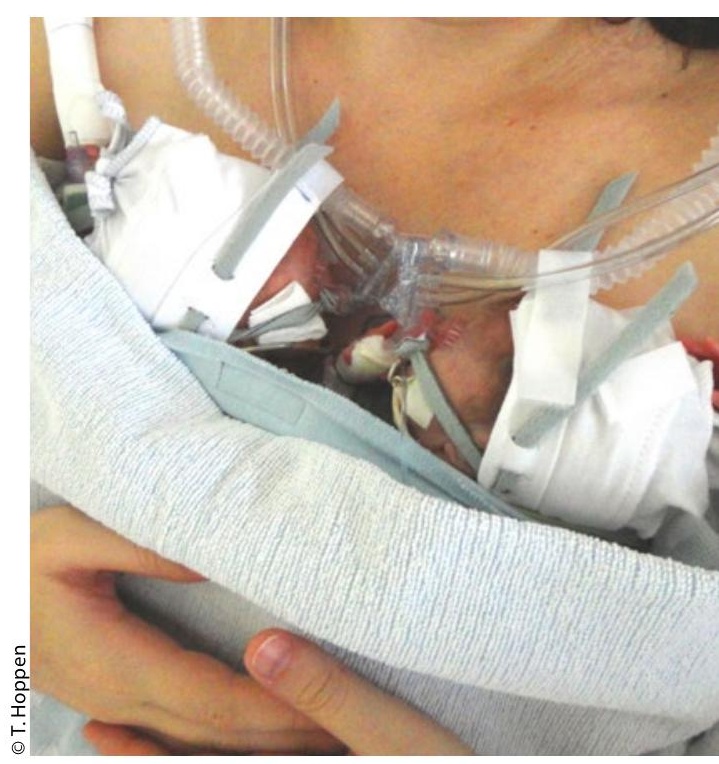

Vorsicht: hier kann es zum EKG-Alarm kommen!

ring während des Kanguruhings darstellt. Alternativ können auch EKG-Elektroden auf dem Rücken des Kindes platziert werden.

Dr. Thomas Hoppen

\section{Gibt es einen „ungefährlichen“ Blutzuckerspiegel beim Neugeborenen?}

Seit Jahrzehnten ist die Problematik der neonatalen Hypoglykämie wissenschaftlich umstritten. Niemand weiß bislang, wie niedrig ein Blutzuckerspiegel sein kann und wie lange er bestehen muss, bis ein Schaden auftritt. In einer Studie wurde das neurologisches Outcome bei zwei Jahre alten Kindern untersucht, bei denen als Neugeborene eine Hypoglykämie aufgetreten war.

mmer wieder wird darauf hingewiesen, dass die in den Leitlinien angegebenen Grenzwerte für Blutzuckerspiegel von Neugeborenen nicht wissenschaftlich fundiert sind. Die Arbeitsgruppe von Chris McKinlay aus Auckland, Neuseeland, hat sich dieser Frage nun mit einem ganz neuen Ansatz gewidmet. In ihrer prospektiven Studie untersuchte sie 404 Neugeborene mit einem Gestationsalter von wenigstens 35 Wochen, bei denen ein Risiko für eine Hypoglykämie bestand. Ihr Ziel war es, den Blutzuckerspiegel in den ersten 48 Lebensstunden nach Möglichkeit über $47 \mathrm{mg} /$ dl $(2,6 \mathrm{mmol} / \mathrm{l}) \mathrm{zu}$ halten. Dabei hatten die Wissenschaflter in konventioneller Weise punktuelle Blutzuckeruntersuchungen vorgenommen, aber unabhängig davon zusätzlich den Blutzucker kontinuierlich mit subkutan eingebrachten Sensoren gemessen. Die Ergebnisse der letztgenannten Messung waren den behandelnden Schwestern und Ärzten nicht bekannt.

Erwartungsgemäß ergaben sich bei einem Teil der Kinder mit den punktuellen Blutzuckermessungen Werte unter 47 mg/dl: Bei $53 \%$ der Probanden kam es zu einer neonatalen Hypoglykämie.
Alle Kinder mit entdeckten Hypoglykämien wurden dann nach den üblichen Standards behandelt. Zusätzlich wurde beobachtet, das etwa ein Viertel der Kinder, die durch die punktuellen Blutzuckermessungen nicht aufgefallen waren, bei der kontinuierlichen Messung hypoglykämische Werte zeigten.

Alle Kinder wurden im Alter von zwei Jahren einer differenzierten neurologischen Nachuntersuchung unterzogen. Sie wurden unter anderem mit dem Bayley Scales of Infant Development (BSIDIII) untersucht. Das Risiko für neurologische Defizite war bei den Kindern mit neonatalen Hypoglykämien nicht gröBer als in der Gruppe, deren Blutzuckerwerte immer über $47 \mathrm{mg} / \mathrm{dl}$ gelegen hatte. Dies galt auch für Neugeborene, bei denen mehrere oder schwere Episoden von Hypoglykämie aufgetreten waren. Auch die Neugeborenen, die lediglich bei der kontinuierlichen Messung unter den Grenzwert gefallen und deshalb nicht behandelt worden waren, zeigten später keine neurologischen Defizite. 